\title{
Terapia nutricional no paciente adulto em oxigenação por membrana extracorpórea: revisão de literatura
}

\section{Nutrition therapy of adult patient during extracorporeal membrane oxygenation: a literature review}

\section{DOI: 10.37111/braspeni.2020352011}

Liane Murari Rocha'

Mayumi Shima²

Branca Jardini de Freitas ${ }^{3}$

Silvia Maria Fraga Piovacari ${ }^{4}$

\section{Unitermos:}

Oxigenação por membrana extracorpórea. Terapia nutricional. Necessidades nutricionais.

\section{Keywords:}

Extracorporeal membrane oxygenation. Nutrition therapy. Nutritional requirements.

\section{Endereço de correspondência:}

Silvia Maria Fraga Piovacari

Departamento de Nutrição Clínica do Hospital Israelita Albert Einstein

Av. Albert Einstein, 627 - São Paulo, SP, Brasil - CEP. 05651-901

E-mail: silvia.piovacari@einstein.br

\section{Submissão}

5 de junho de 2020

\section{Aceito para publicação}

22 de junho de 2020

\begin{abstract}
RESUMO
Introdução: A oxigenação por membrana extracorpórea (ECMO) fornece suporte cardiopulmonar parcial ou total a pacientes com falência cardíaca ou pulmonar grave, aguda e reversível. Apesar do crescente uso da técnica em pacientes adultos, pouco se sabe sobre os cuidados nutricionais. Objetivou-se investigar dados disponíveis sobre o suporte nutricional de pacientes adultos em uso da ECMO, visando auxiliar na elaboração de protocolos institucionais. Método: Foi realizada uma revisão narrativa da literatura, evidenciando o manejo do suporte nutricional, razões da baixa adequação nutricional, necessidades nutricionais e o papel da nutrição nas complicações da ECMO. Resultados: As estratégias de pesquisa identificaram estudos publicados até 11 de outubro de 2019. Apenas estudos primários indexados nas bases de dados MEDLINE, Scopus e Web of Science foram avaliados, resultando na inclusão de 11 estudos. Há evidências de que a nutrição enteral precoce é segura e bem tolerada, podendo ser iniciada por via gástrica. A disfunção gastrointestinal com altos volumes residuais gástricos é um achado comum, no entanto, existem evidências positivas do tratamento com drogas procinéticas, havendo ainda como alternativa o uso de sonda nasojejunal e nutrição parenteral. É possível atingir a adequação nutricional $(>80 \%)$ que está associada a menor mortalidade, entretanto, a subnutrição é frequente, entre os principais motivos está a interrupção da dieta para procedimentos terapêuticos/diagnósticos. A nutrição parenteral suplementar é uma estratégia importante, porém exige cuidado especial com a administração de glicose e gorduras. Os dados sobre as necessidades nutricionais são controversos, assim como as diferenças no suporte nutricional e nas intercorrências entre os tipos de ECMO (VA e VV). Conclusão: Apesar da maioria dos estudos ser observacional retrospectivo, as evidências demonstram que a nutrição é segura, bem tolerada e está associada a menor mortalidade dos pacientes em ECMO.
\end{abstract}

\section{ABSTRACT}

Introduction: Extracorporeal membrane oxygenation (ECMO) provides partial or total cardiopulmonary support to patients with severe, acute and reversible cardiac or pulmonary failure. Despite the increasing use of the technique in adult patients, information on nutritional care is scarce. The objective was to investigate available data on the nutritional support of adult patients using ECMO in order to assist in the development of institutional protocols. Methods: A narrative review of the literature was led in order to showing the management of nutritional support, reasons for low nutritional adequacy, nutritional needs and the role of nutrition in ECMO complications. Results: Search strategies identified studies published up to October $11^{\text {th }}, 2019$. Only primary studies, indexed in the MEDLINE, Scopus and Web of Science data bases were evaluated, resulting in the inclusion of 11 studies. There is evidence that early enteral nutrition is safe and well tolerated and can be initiated via the gastric route. Gastrointestinal dysfunction with high gastric residual volumes is a common finding. However, there is positive evidence of treatment with prokinetic drugs, with the option of using a nasojejunal tube and parenteral nutrition. It is possible to achieve nutritional adequacy ( $>$ $80 \%$ ), which is associated with lower mortality. However, malnutrition is recurrent, among the main reasons is the interruption of the diet for therapeutic/diagnostic procedures. Supplemental parenteral nutrition is an important strategy, but it requires special care with the associated administration of glucose and fats. Data on nutritional needs are controversial, as well as the differences in nutritional support and intercurrences between types of ECMO (VA and V). Conclusion: It is concluded that although most studies are observational retrospective, the evidence shows that nutrition is safe, well tolerated and is associated with lower mortality of patients on ECMO.

1. Nutricionista. Especialista em Nutrição Hospitalar pela Faculdade Israelita de Ciências da Saúde Albert Einstein. Mestre em Alimentos e Nutrição e doutora em Ciências na área de Clínica Médica pela Universidade Estadual de Campinas (Unicamp). Nutricionista Clínica do Hospital Israelita Albert Einstein, São Paulo, SP, Brasil.

2. Nutricionista. Especialista em Nutrição Clínica pela Associação Brasileira de Nutrição - ASBRAN e pela Divisão de Nutrição e Dietética do Instituto Central do Hospital das Clinicas - FMUSP. Especialista em Nutrição Parenteral e Enteral pela Sociedade Brasileira de Nutrição Parenteral e Enteral - SBNPE/BRASPEN. Mestre em Ciências da Saúde pela Faculdade Israelita de Ciências da Saúde Albert Einstein. Nutricionista Sênior do Departamento de Pacientes Graves do Hospital Israelita Albert Einstein.

3. Nutricionista. Especialista em Nutrição Parenteral e Enteral pela Sociedade Brasileira de Nutrição Parenteral e Enteral -SBNPE/BRASPEN. Residência em Nutrição Hospitalar pela Pontifícia Universidade Católica de Campinas - PUC -Campinas. Pós Graduação em Gestão em Saúde -Faculdade Getúlio Vargas. Nutricionista Clínica do Departamento de Pacientes Graves do Hospital Israelita Albert Einstein.

5. Nutricionista. Especialista em Nutrição Clínica pela Associação Brasileira de Nutrição - ASBRAN e pelo Centro Universitário São Camilo. Especialista em Nutrição Parenteral e Enteral pela Sociedade Brasileira de Nutrição Parenteral e Enteral - SBNPE/BRASPEN. MBA Executivo em Gestão de Saúde - INSPER com extensão internacional em Barcelona - Espanha. Mestranda em Ensino em Saúde pela Faculdade Israelita de Ciências da Saúde Albert Einstein. Coordenadora de Nutrição Clínica do Hospital Israelita Albert Einstein. Coordenadora da Pós-graduação em Nutrição Hospitalar - Faculdade Israelita de Ciências da Saúde Albert Einstein. 


\section{INTRODUÇÃO}

A oxigenação por membrana extracorpórea, do inglês extracorporeal membrane oxygenation (ECMO), ou suporte extracorpóreo de vida - extracorporeal life support (ECLS) é um modelo invasivo idealizado para fornecer suporte cardiopulmonar parcial ou total a pacientes com falência cardíaca ou pulmonar grave, aguda e reversível, com alto risco de mortalidade que não respondem às intervenções terapêuticas convencionais. Pode ser utilizada de pacientes neonatais a adultos. $\bigcirc$ mecanismo consiste na drenagem de sangue venoso e seu bombeamento por meio de uma membrana em que é adicionado oxigênio $\left(\mathrm{O}_{2}\right)$ e retirado gás carbônico $\left(\mathrm{CO}_{2}\right)$. Após, o sangue oxigenado é devolvido ao paciente pela circulação venosa (venovenosa - W) ou arterial (venoarterial - VA). A ECMO VA é indicada como suporte cardíaco nos pacientes com função pulmonar preservada ou não. A ECMO W é indicada para casos de insuficiência respiratória com função cardíaca preservada. Entretanto, há certa dificuldade para a elegibilidade dos pacientes para ECMO por existirem poucos critérios de indicação e de contraindicação absolutas ${ }^{1-3}$.

Devido a sua complexidade, o suporte por meio da ECMO exige uma equipe multiprofissional treinada, tem um custo elevado, porém com uma relação de custo-utilidade aceitável. Deste modo, nos últimos anos, tem ocorrido aumento no número de centros que realizam ECMO. Um dado parcial do primeiro semestre de 2019 da Extracorporeal Life Support Organization (ELSO) americana demonstra um elevado número de casos de ECMO, sendo 21.874 pulmonares e 22.193 cardíacos $^{4,5}$.

Os pacientes em ECMO são considerados pacientes graves, que têm como característica atividade metabólica elevada, catabolismo de proteínas e balanço nitrogenado negativo. A adequada oferta de energia e proteína está associada a melhores desfechos clínicos ${ }^{6}$. Com relação à nutrição dos pacientes adultos em ECMO, os dados são escassos, a experiência multicêntrica coletiva ainda é um importante recurso de informação para a equipe multidisciplinar, pois muitas questões permanecem sem resposta e consenso ${ }^{7,8}$. Até 0 momento, há poucas diretrizes, todas publicadas recentemente, mas sem informações específicas aos pacientes em ECMO9,10. Somente a diretriz da German Society for Nutritional Medicine (DGEM) ${ }^{11}$ sobre nutrição clínica em medicina intensiva tem uma seção destinada a grupos de pacientes específicos, que incluí aqueles em ECMO. São abordadas a forma de determinação do gasto energético, a via preferencial de nutrição (enteral ou parenteral), quantidade de gordura e como deve ser administrada a nutrição parenteral (NP). Apesar disso, outros detalhes precisam ser elucidados considerando a necessidade dos cuidados na prática clínica, como manejo do suporte nutricional, razões da baixa adequação nutricional, necessidades nutricionais, inclusive com o uso concomitante de terapia de substituição renal, e o papel da nutrição nas complicações da ECMO, como a formação de coágulos.

Considerando o crescente uso da técnica de suporte extracorpóreo e visando a uma abordagem interdisciplinar com suporte nutricional adequado, almejando melhores resultados a longo prazo, este trabalho tem por objetivo investigar dados disponíveis sobre o suporte nutricional, a fim de propor um protocolo de conduta nutricional a pacientes adultos em uso da ECMO, em unidade de terapia intensiva (UTI).

\section{MÉTODO}

O presente estudo consiste em revisão narrativa da literatura. Foram realizadas buscas nas bases de dados MEDLINE (via Pubmed), Scopus e Web of Science, em 11 de outubro de 2019, sem restrição de tempo. As listas de referências dos artigos incluídos também foram consultadas. Somente artigos publicados em inglês e português foram incluídos.

A revisão incluiu estudos primários retrospectivos e prospectivos realizados em seres humanos e foram utilizadas as seguintes palavras-chave: extracorporeal membrane oxygenation, nutrition therapy, nutritional support, nutrition requirements, nutrition assessment, enteral nutrition, protein, energy, energy expenditure, calorimetry, metabolism. Para o cruzamento dos descritores foram utilizados os operadores booleanos "OR" e "AND", com o objetivo de localizar uniões e intersecções de termos, ampliando ou especificando a informação desejada.

Os estudos identificados nas buscas foram importados para o software Endnote Web ${ }^{\circledR}$, para remoção de quaisquer duplicatas. Inicialmente, os títulos e resumos foram selecionados e os artigos relevantes foram eleitos para uma leitura completa do texto. Os critérios de exclusão foram estudos com animais, testes com drogas, estudos com crianças/ adolescentes e revisões. A extração de dados foi realizada de forma independente e incluiu informações sobre autores, ano, idade, tipo de estudo, delineamento e manejo nutricional. Foi realizada síntese narrativa por meio de tabela e resumo.

\section{RESULTADOS}

A busca resultou em 564 artigos, mas, após a remoção dos registros duplicados, 535 artigos permaneceram. $\mathrm{Na}$ sequência foi realizada a triagem dos títulos e resumos, 18 estudos foram considerados potencialmente elegíveis, mas apenas 11 avaliaram nutrição em ECMO. Os artigos que abordam o suporte nutricional são escassos e, em geral, apresentam em comum limitações como delineamento observacional retrospectivo e coleta de dados em um único centro ${ }^{12-17}$. Há apenas dois estudos observacionais 
prospectivos publicados até 0 momento ${ }^{18,19}$, sendo um deles multicêntrico ${ }^{19}$. Os estudos foram realizados incluindo pacientes em ECMO W e VA ${ }^{12,13,16,19}$ somente ECMO VV ${ }^{14,15}$ ou somente ECMO VA ${ }^{17,18}$.

\section{Determinação do Gasto Energético}

A necessidade energética pode ser medida ou estimada por fórmulas. A calorimetria indireta é um método seguro e não invasivo utilizado para medir o gasto energético em repouso. Por apresentar boa acurácia ( $2 \%$ a $5 \%$ de erro) é considerado padrão-ouro. Este método estima, por meio de um equipamento específico, o calor gerado pelo organismo a partir da mensuração do $\mathrm{O}_{2}$ consumido, do $\mathrm{CO}_{2}$ produzido e do nitrogênio urinário excretado em um determinado período. Após, esses valores são incluídos na equação de Weir ${ }^{20}$, para obtenção do valor do gasto energético ${ }^{21}$. A diretriz sobre nutrição clínica em UTI da European Society for Clinical Nutrition and Metabolism (ESPEN) recomenda o uso da calorimetria indireta na prática clínica para determinação da necessidade energética ${ }^{10}$. Entretanto, o uso da calorimetria indireta em pacientes em ECMO tem como limitação a captação de $\mathrm{O}_{2}$ e remoção de $\mathrm{CO}_{2}$ serem divididas entre a ventilação mecânica e o pulmão artificial. Com o propósito de viabilizar o uso da calorimetria indireta em pacientes em ECMO, estudo realizado com um paciente propôs um modelo teórico baseado em uma medição da calorimetria em dois níveis, pulmão do paciente e artificial ${ }^{22}$, e a inserção das medições obtidas na equação de Weir modificada ${ }^{20}$.

Alguns anos depois, a calorimetria indireta foi utilizada para avaliar o gasto energético de 20 pacientes com síndrome do desconforto respiratório agudo (SDRA) em ECMO W comparados a 20 pacientes sem ECMO. Para isso, os autores desenvolveram um protocolo específico intitulado MEEP, do inglês Measuring Energy Expenditure in ECMO Patients, que considerou um ponto adicional de troca gasosa ao lado dos pulmões, utilizando para o cálculo a captação de $\mathrm{O}_{2}$ e eliminação de $\mathrm{CO}_{2}$ pela membrana da ECMO. Portanto, os dois locais de troca gasosa foram medidos separadamente e, posteriormente, foram combinados antes do uso da equação de Weir ${ }^{20}$, para o cálculo do gasto energético. Também foram coletadas amostras de sangue para análise dos gases sanguíneos pré e pós-membrana de troca gasosa, para medir as trocas gasosas da ECMO. Os resultados demonstraram que o protocolo MEEP é viável e que os valores não diferiram entre pacientes com SDRA em ECMO e sem ECMO. Os autores ainda testaram equações preditivas e nenhuma delas correspondeu ao gasto energético medido ${ }^{23}$. Estudo com objetivo similar realizado com 7 pacientes demonstrou dados que corroboram com o estudo anterior, o gasto energético calculado forneceu informações inadequadas, sendo a calorimetria o método padrão-ouro viável aos pacientes em ECMO W24.
Apesar da realização destes estudos exploratórios sobre - uso da calorimetria indireta em pacientes em ECMO, há algumas limitações importantes, como o tamanho das amostras, havendo a necessidade de estudos maiores para validação com amostras representativas. Por esta razão e pela dificuldade da realização da calorimetria indireta na prática clínica, a DGEM recomenda o uso de fórmulas baseadas no peso para estimar a necessidade energética destes pacientes ${ }^{11}$.

\section{Necessidades Nutricionais}

Até o momento, não há uma recomendação calórica e proteica específica para pacientes em ECMO. Nos estudos publicados (Tabela 1), o cálculo da necessidade calórica foi realizado pela equação de Schofield ${ }^{25}$, com diferentes fatores de estresse ${ }^{12,13}$, por fórmula de bolso ${ }^{14,26}$ e equação de Harris-Benedict ${ }^{16,27}$. Para necessidade proteica, os estudos utilizaram como valor mínimo $1,2 \mathrm{~g} / \mathrm{kg} / \mathrm{dia}^{12-16,18} \mathrm{e}$, como valor máximo, 1,5 g/kg/dia' ${ }^{12,15}$.

Conforme demonstrado, o gasto energético de pacientes graves em ECMO e sem ECMO não diferiu, reforçando, portanto, que as recomendações para pacientes graves são aplicáveis a estes pacientes ${ }^{23}$. A diretriz da ESPEN ${ }^{10}$ sobre nutrição clínica em UTI menciona que, na impossibilidade de realização de calorimetria, como no caso dos pacientes em ECMO, equações simples como a fórmula de bolso podem ser utilizadas. Recomendam-se os valores: 20 e $25 \mathrm{kcal} /$ $\mathrm{kg} /$ dia. Para pacientes obesos, o cálculo da necessidade energética pode ser feito utilizando o peso corporal ajustado. A DGEM ${ }^{11}$ desestimula o uso de fórmulas complexas e também recomenda utilizar fórmula de bolso: $24 \mathrm{kcal} / \mathrm{kg}$. Já a diretriz da Society of Critical Care Medicine (SCCM) e da American Society for Parenteral and Enteral Nutrition (A.S.P.E.N.) ${ }^{28}$ recomenda utilizar de 25 a $30 \mathrm{kcal} / \mathrm{kg}$. Nota-se na tabela 1 que os estudos utilizaram formas muito distintas para estimar a necessidade calórica, havendo a necessidade de uma maior padronização de acordo com as recomendações das diretrizes, para que a experiência da prática clínica contribua na determinação de valores calóricos adequados aos pacientes em ECMO.

Por outro lado, os valores de proteínas utilizados foram semelhantes às recomendações das sociedades ${ }^{12-14,16}$. A recomendação da $\operatorname{ESPEN}^{10}$ durante a doença grave é de 1,3 $\mathrm{g} / \mathrm{kg} /$ dia, com aumento progressivo. A DGEM ${ }^{11}$ recomenda utilizar o peso atual no cálculo das necessidades proteicas dos pacientes não obesos, com índice de massa corporal (IMC) $<30 \mathrm{~kg} / \mathrm{m}^{2}$, e utilizar entre 1,0 e 1,2 g/ $/ \mathrm{kg} /$ dia de proteína, iniciando na fase aguda oferecendo até 75\% da meta e 100\% da meta pós-fase aguda. Já a diretriz da SCCM e A.S.P.E.N. ${ }^{28}$ recomenda fornecer de 1,2 a 2,0 g/ $/ \mathrm{kg} /$ dia de peso atual. Ainda com relação às proteínas, um estudo realizado com o objetivo de investigar o catabolismo proteico durante 
Tabela 1 - Necessidades nutricionais dos pacientes de acordo com o modo de ECMO (VV e/ou VA) dos estudos incluídos na revisão.

\begin{tabular}{|c|c|c|c|c|c|}
\hline Autores, ano & Delineamento & $\begin{array}{l}\text { Participantes/ } \\
\text { idade }\end{array}$ & $\begin{array}{l}\text { Tipo de } \\
\text { ECMO }\end{array}$ & $\begin{array}{l}\text { Necessidade } \\
\text { calórica }\end{array}$ & Necessidade proteica \\
\hline Ferrie et al. ${ }^{13}, 2013$ & $\begin{array}{l}\text { Observacional } \\
\text { retrospectivo }\end{array}$ & $\begin{array}{c}86 \text { pacientes } \\
\text { Média 44,4 anos (DP 15,9) }\end{array}$ & $\begin{array}{l}\text { VA: } 31 \text { pacientes } \\
\text { VV: } 55 \text { pacientes }\end{array}$ & $\begin{array}{l}\text { Equação de Schofield²5, } \\
\text { fator de estresse } 1,1 \text { a 1,2 }\end{array}$ & Mínimo 1,2 g/kg/dia \\
\hline Lu et al. ${ }^{16}, 2018$ & $\begin{array}{l}\text { Observacional } \\
\text { retrospectivo }\end{array}$ & $\begin{array}{l}102 \text { pacientes ( } 70 \text { homens) } \\
\text { Média } 55,4 \text { anos (DP } 17,4)\end{array}$ & $\begin{array}{l}\text { VA: } 77 \text { pacientes } \\
\text { VV: } 25 \text { pacientes }\end{array}$ & $\begin{array}{l}\text { Equação de Harris-Benedict, } \\
\text { fator atividade } 1,2 \text { e fator } \\
\text { estresse } 1,2 \text { a } 1,5\end{array}$ & Mínimo 1,2 g/kg/dia \\
\hline MacGowan et al. ${ }^{14}, 2019$ & $\begin{array}{l}\text { Observacional } \\
\text { retrospectivo }\end{array}$ & $\begin{array}{c}203 \text { pacientes } \\
\text { Mediana } 44 \text { anos (IQ 33-35) }\end{array}$ & $\begin{array}{l}\text { VV: } 203 \text { pacien- } \\
\text { tes }\end{array}$ & 25 a $30 \mathrm{kcal} / \mathrm{kg} / \mathrm{dia}$ & Mínimo 1,2 g/kg/dia \\
\hline Scott et al. ${ }^{15}, 2004$ & $\begin{array}{l}\text { Observacional } \\
\text { retrospectivo }\end{array}$ & $\begin{array}{l}27 \text { pacientes } \\
\geq 18 \text { anos }\end{array}$ & V: 27 pacientes & $25 \mathrm{kcal} / \mathrm{kg}$ & 1,2 a $1,5 \mathrm{~g} / \mathrm{kg} / \mathrm{dia}$ \\
\hline Umezawa et al. ${ }^{18}, 2013$ & $\begin{array}{l}\text { Observacional } \\
\text { retrospectivo }\end{array}$ & $\begin{array}{c}7 \text { pacientes } \\
\text { Idade não informada }\end{array}$ & VA: 7 pacientes & $25 \mathrm{kcal} / \mathrm{kg}$ & Não informado \\
\hline
\end{tabular}

$\mathrm{DP}=$ desvio padrão; $\mathrm{ECMO}=$ Extracorporeal membrane oxygenation; $\mathrm{IQ}=$ intervalo interquartil; $\mathrm{VA}=$ venoarterial; $\mathrm{W}=$ venovenoso

o suporte da ECMO W sugeriu que o estado nutricional dos pacientes pode influenciar no balanço nitrogenado. $O$ resultado do balanço nitrogenado demonstrou que pacientes não obesos tiveram menor catabolismo do que pacientes com obesidade, respectivamente $-1,7 \pm 5,7$ vs. $-11,5 \pm$ $9,6^{29}$. Entretanto, tratou-se de estudo observacional com amostra pequena, portanto, são necessários mais estudos para validar este dado.

\section{Terapia Nutricional}

\section{Nutrição Enteral ou Parenteral}

Há uma grande preocupação quanto à segurança da nutrição do paciente em ECMO, considerando os efeitos das alterações hemodinâmicas, como a redução da perfusão intestinal, que se dá pela diminuição do fluxo pulsátil para a microcirculação. Estas alterações são mais comuns nos pacientes em ECMO VA, pois na ECMO W o fluxo pulsátil é melhor preservado. Entretanto, a ativação da inflamação sistêmica pode causar disfunção intestinal nos dois modos de ECMO, por provocar disfunção da barreira intestinal e permitir a translocação bacteriana ${ }^{30,31}$. Algumas condições clínicas também podem prejudicar a função intestinal, como hipóxia prolongada e estado de baixo débito cardíaco por vários dias. $\bigcirc$ uso de grandes volumes de líquido intravenoso na ECMO W para manter altas taxas de fluxo podem causar edema generalizado, que pode envolver o intestino e reduzir a absorção e a motilidade ${ }^{13}$. Além disso, estes pacientes estão entre os mais gravemente enfermos e são mais propensos a uma permanência prolongada na UTI ${ }^{32}$.

Apesar das alegações que as alterações hemodinâmicas resultantes podem tornar a nutrição enteral (NE) insegura ou mal tolerada, foi demonstrado que a NE pode ser bem tolerada em pacientes em ECMO W e $\mathrm{VA}^{13}$, inclusive naqueles com insuficiência hemodinâmica grave em ECMO VA, ainda que a amostra tenha sido pequena, tratou-se de estudo prospectivo e não foram atribuídos eventos adversos graves com relação à $N E^{18}$.

De modo geral, a NE foi extensamente utilizada nos estudos, atingindo $96 \%$ e, até mesmo, $100 \%$ dos pacientes ${ }^{13,15-18}$, sendo, portanto, a via preferencial para nutrição. A NP exclusiva foi utilizada por apenas $0,3 \%$ a $4 \%$ dos pacientes ${ }^{12,15}$. Estudo multicêntrico realizado em 8 UTIs da Austrália e Nova Zelândia observou que a NE foi o modo de nutrição mais utilizado, sendo fornecida em $84 \%$ dos dias ${ }^{19}$.

A investigação da tolerância da $N E$, via sonda nasogástrica, em pacientes em ECMO W e VA, demonstrou que 38\% dos pacientes apresentaram intolerância alimentar durante os 5 primeiros dias, destes $60,6 \%$ foram tratados, de forma efetiva, com drogas procinéticas, sem haver a necessidade de nutrição pós-pilórica a nenhum paciente. Entretanto, 
é importante considerar o número reduzido de pacientes (36\%) em ECMO VA incluídos neste estudo, modo em que as alterações hemodinâmicas reduzem mais o fluxo pulsátil e, portanto, a perfusão intestinal ${ }^{13}$.

Outros estudos realizados com pacientes em ECMO VA investigaram eventos adversos da NE. Estudo observacional prospectivo multicêntrico realizado com ambos os modos de ECMO, sendo $61 \%$ pacientes em ECMO VA, relatou a não tolerância de NE em $37 \%$, ocorrência de sangramento gastrointestinal em $24 \%$ e isquemia intestinal em 4,5\% da amostra total. Devido ao uso de agentes anticoagulantes, o sangramento gastrointestinal não é um achado imprevisto e, apesar da presença destes eventos adversos, o uso de NP foi relativamente baixo ( $7 \%$ dos dias), sendo a NE a forma de nutrição mais utilizada ${ }^{19}$. Houve o relato de ocorrência de isquemia intestinal menor (1\%) em estudo observacional retrospectivo e aconteceu no grupo que recebeu NE tardiamente (após 3 dias). Não houve nenhum caso no grupo que recebeu NE em até 2 dias. Entretanto, futuras análises são necessárias para investigar a associação entre isquemia intestinal e início tardio da $\mathrm{NE}^{17}$. Com relação à isquemia intestinal e suas consequências, esta não foi um fator que contribuiu com os $29,2 \%$ de mortes de pacientes em ECMO W e VA, apesar da sua relevância na piora do quadro clínico ${ }^{12}$. A nutrição, inclusive, é um fator relacionado à sobrevida dos pacientes, sendo a maior adequação nutricional fortemente associada à menor taxa de mortalidade ${ }^{16}$.

No que se refere às diretrizes, de fato, a DGEM recomenda o uso de NE nos pacientes críticos em ECMO V e $V A$, que pode ser utilizada em todas as fases da doença, desde que haja ausência de sinais de disfunção intestinal grave e/ou instabilidade hemodinâmica. A tolerância metabólica deve ser monitorada de perto ${ }^{11}$. Com relação ao uso de drogas vasoativas, não é um impeditivo, conforme já demonstrado na literatura. A administração de NE via sonda com posição gástrica concomitante ao uso de doses baixas e estáveis de vasopressores com um monitoramento rigoroso de sinais de intolerância ou agravamento da estabilidade hemodinâmica apresenta um baixo risco de necrose intestinal ${ }^{33}$.

\section{Nutrição Enteral Precoce}

A única menção específica que a diretriz da ESPEN ${ }^{10} \mathrm{faz}$ sobre pacientes em ECMO é a recomendação do uso de NE precoce, iniciada entre 24 e 48 h após a admissão, também indicada pela DGEM ${ }^{11}$, considerando a ausência de sinais de disfunção intestinal grave e/ou instabilidade hemodinâmica. Há evidência de que a NE precoce é benéfica, sendo considerada também ao menos uma dose trófica, importante por contribuir para a manutenção da barreira intestinal e da imunidade intestinal $^{33,34}$.
De fato, a NE precoce se demonstrou ser segura e bem tolerada, sendo iniciada após 13,1 $\pm 16,7 \mathrm{~h}$ em um estudo $^{13}$, em 13,5 horas (IQ, 9,0 e 23,5) em um outro estudo $^{14}$, com $96,6 \%$ dos pacientes tendo recebido suporte nutricional em 48 horas. Outros estudos também reportaram que a NE precoce é segura em adultos recebendo suporte em ECMO, não sendo atribuída a eventos adversos graves, como isquemia intestinal, sangramento gastrointestinal ou outras complicações ${ }^{15,18}$.

Além disso, o tempo para o início do suporte nutricional não influenciou a sobrevida em 6 meses dos pacientes em ECMO W ${ }^{14}$ e foi significativamente associada a menor mortalidade em pacientes em ECMO VA com choque cardiogênico ${ }^{17}$. Ainda com relação à sobrevida, estudo demonstrou que a NE precoce é segura e bem tolerada, não havendo diferença no início da dieta entre os sobreviventes e não sobreviventes ${ }^{16}$.

\section{Vias de Acesso e Métodos de Administração da Nutrição Enteral}

Em geral, os pacientes em ECMO dos estudos incluídos iniciaram o suporte nutricional de acordo com o protocolo da UTI, que defende o início da alimentação gástrica após 24 a 48 h da admissão. Por esta razão, o uso de sonda nasogástrica foi extensamente relatado ${ }^{13-15,18,19}$. Em alguns estudos, não houve a necessidade de sonda nasojejunal em nenhum paciente, sendo a sonda nasogástrica utilizada por todos os pacientes com NE ${ }^{12,13,15,18}$. O calibre das sondas utilizadas foi de 8 a $12 \mathrm{Fr}^{18}$ e de 16 a $18 \mathrm{Fr}^{15}$

O uso de sonda jejunal foi relatado em $6 \%$ a $34,5 \%$ dos pacientes $^{12,14}$ e realizado em $10 \%$ dos dias nos pacientes em ECMO VA e $\mathrm{W}^{19}$. O principal critério para utilização da sonda na posição pós-pilórica foi a intolerância gastroinstestinal. Porém, a primeira recomendação era o uso de agentes procinéticos e, na persistência da intolerância, como segunda alternativa, recomendava-se a mudança da posição da sonda para jejunal. Na ausência de melhora, considerava-se como última alternativa o uso de NP12-14.

Com relação à administração da dieta, o método contínuo foi o mais relatado ${ }^{12,15,18}$, iniciando com a velocidade de infusão de $20 \mathrm{ml} / \mathrm{h}^{15,18}$. Um estudo utilizou bolus, tolerado por $73 \%$ dos pacientes e, nos casos de intolerância ao bolus, foi utilizada NE contínua, em $27 \%$ dos pacientes ${ }^{16}$.

\section{Fórmulas Enterais}

A escolha da fórmula enteral apropriada ao paciente requer a avaliação da capacidade de digestão e absorção. De um modo geral, a escolha das fórmulas utilizadas nos estudos foi baseada no protocolo da UTI, considerando a grande maioria dos estudos ser de natureza retrospectiva. Os dados quanto à fórmula enteral foram divergentes, houve o relato do uso de fórmula padrão polimérica isocalórica, sem 
o uso de imunonutrição ${ }^{12}$; uso de fórmula isocalórica padrão, tolerada por $78 \%$ dos pacientes, sendo utilizada fórmula oligomérica nos demais $22 \%{ }^{16}$; uso de fórmula oligomérica e de fórmula de imunonutrição, com arginina e glutamina em $42,3 \%$ dos pacientes ${ }^{15}$; e, ainda, o uso de fórmula polimérica isocalórica com fibras para iniciar, fórmula de imunonutrição como manutenção e, nos casos de diarreia, uso de fórmula hipercalórica com fibras solúveis ${ }^{18}$. Um estudo apresentou a densidade calórica das fórmulas, sendo comumente utilizadas fórmulas de 1,25 a 2,0 kcal $/ \mathrm{ml}$ ( $80 \%$ dos pacientes). Fórmulas com $1,0 \mathrm{kcal} / \mathrm{ml}$ eram muito pouco utilizadas ${ }^{19}$. A escolha da fórmula mais adequada é de suma importância, considerando a intolerância gastrointestinal, comum nestes pacientes, e a relevância da adequada oferta de calorias e proteínas. $\bigcirc$ uso de fórmula hiperproteica, por exemplo, pode influenciar na melhor adequação nutricional, conforme relatado ${ }^{14}$.

Com relação aos líquidos, o uso de grandes volumes durante a ECMO W para manter as altas taxas de fluxo resulta em edema generalizado. $O$ efeito do volume extra de fluido faz com que haja necessidade de restrição de outros fluidos, incluindo a nutrição. Houve o relato da prescrição de restrição hídrica a $77,6 \%$ dos pacientes. Portanto, a escolha da fórmula enteral fica limitada aos produtos de baixo volume disponíveis. Na escolha da fórmula enteral, deve-se levar em consideração também o uso de medicamentos que são administrados com soro glicosado e/ou medicamentos como propofol, que possuem lipídeos em sua fórmula, contendo calorias que devem ser contabilizadas. Foi observado que o propofol contribuiu significativamente para o aporte calórico, exigindo uma redução da nutrição em $81,6 \%$ dos pacientes, fornecendo mais de $400 \mathrm{kcal}$ em $24 \mathrm{~h}$ a $37,2 \%$ deles. Entretanto, a média foi de $130 \pm 124$ kcal fornecidas pelo propofol em pacientes em ECMO V e VA ${ }^{13}$.

\section{Nutrição Parenteral}

A NP foi empregada na presença de intolerância à NE, constatada pela presença de alto volume residual gástrico (VRG), presença de sangramento intestinal, isquemia intestinal, entre outros ${ }^{19}$. Nos casos de intolerância à dieta, conforme mencionado, a NP foi utilizada em última alternativa, após a tentativa do uso de agentes procinéticos, seguido pela sonda na posição pós-pilórica ${ }^{12-14}$. O uso da NP foi relativamente baixo, sendo relatada a utilização em $7 \%$ dos dias ${ }^{19}$. Em um estudo, 20,9\% dos pacientes receberam NP para substituir ou suplementar a NE mal tolerada. Destes, 55,6\% tiveram a NE reiniciada como única forma de nutrição em 4 dias $^{13}$.

No que se refere à intolerância da NE, o critério utilizado para avaliá-la foi a infusão de $<50 \%$ do volume durante $24 \mathrm{~h}$; $30,8 \%$ dos pacientes necessitaram de suporte parcial de NP. O objetivo calórico calculado da NP foi a diferença entre a meta calórica e a infusão de NE. A NP foi descontinuada quando a tolerância à dieta enteral foi $>75 \%$ em $24 \mathrm{~h}$. Houve o relato de um paciente $(3,7 \%)$ com NP exclusiva ${ }^{15}$.

Em outro estudo, a NP suplementar em conjunto com a NE foi utilizada em $25 \%$ dos pacientes. Esta é uma estratégia importante, porém é válido considerar que os pacientes são imunossuprimidos e o acesso central predispõe a infecções. Por se tratar também de pacientes em estresse, o controle da glicose deve ser rigososo ${ }^{12}$. Com relação às gorduras, uma atenção especial deve ser dada a sua quantidade, para evitar que se depositem nos circuitos da ECMO e aumentem a formação de coágulos sanguíneos ${ }^{35}$. Por este motivo, há a recomendação do uso de lipídeos parenterais, porém, no máximo, $1,5 \mathrm{~g} / \mathrm{kg}$ por dia, levando em consideração o teor de ácido linoleico e ácido $\alpha$-linolênico da emulsão, para evitar a deficiência de ácidos graxos essenciais ${ }^{11}$.

\section{Adequação Nutricional}

A adequação nutricional pode ser definida como a razão entre a nutrição fornecida e a meta nutricional, expressa em porcentagem ${ }^{12}$. Devem ser consideradas as calorias e proteínas advindas da NE, NP, glicose intravenosa (IV) e também as calorias de medicamentos, como propofol. A alimentação pode ser considerada adequada quando a razão fica entre $80 \%$ e $110 \%$ das metas estimadas. Um estudo demonstrou a administração adequada das calorias (mediana 89,8\% - IQ, 80,5 e 96,0\%) e proteínas (mediana $84,7 \%$ - IQ, 74,0 e 96,7\%) durante ECMO $\mathrm{V}^{14}$. Um outro estudo realizado com os dois modos de ECMO $(75,5 \%$ pacientes em ECMO VA) também observou adequação calórica e proteica satisfatórias, respectivamente, $88 \%$ e $97 \%$ e $100 \%{ }^{16}$. Porém, em outros estudos, os resultados foram conflitantes, os pacientes em ECMO W e VA toleraram, em média, 79,7\% da meta diária das calorias e 73\% da meta diária de proteína, nas primeiras 2 semanas em um estudo $^{13}$; e no outro, toleraram $55 \%$ da meta calórica ${ }^{12}$. fato de alguns estudos terem atingido uma boa adequação nutricional traz a reflexão da viabilidade de nutrir adequadamente os pacientes em ECMO, considerado um grande desafio. Entretanto, apesar do bom resultado, a presença de subnutrição foi achado comum e ocorreu em $28,3 \%$ dos dias de suporte nutricional ${ }^{14}$.

Entre os principais motivos para a adequação nutricional insuficiente estão as interrupções da nutrição, que atingiram $90,6 \%$ dos pacientes ao menos uma vez, e $46,8 \%$, mais de uma vez, com duração mediana de $9 \mathrm{~h}(\mathrm{IQ}, 3,0 \text { e } 32,0)^{14}$. Quanto ao tempo, houve o relato da interrupção em 53\% dos dias, com duração mediana de 8 h (IQ 4,0 e 15,0) ${ }^{19}$. A interrupção para procedimentos terapêuticos/diagnósticos foi a primeira causa mencionada em vários estudos ${ }^{12,14,19}$, representando entre $31 \%$ e 39,1\% ${ }^{14,19}$, seguido por intolerância 
gastrointestinal/elevados VRG ${ }^{12,14,19}(22 \%$ e 22,8\%) e falta de acesso à NE ou problema relacionado à sonda (14\% e $15,2 \%)^{14,19}$. As demais causas citadas foram: razão desconhecida (12\%), questões no processo de infusão da NE (12\%), a pedido da equipe médica (9\%), preocupações com a tolerância da NE (8\%), jejum para extubação (6\%), entre outros. Com relação às questões no processo de entrega da $\mathrm{NE}$, os comentários foram: "não está na meta", "aumento gradual", "começou tarde", "trocando as bolsas de NE", "admissão tardia" e "aguardando revisão da nutricionista". Todos estes comentários poderiam ser evitados com treinamento adequado da equipe de cuidados aos pacientes em ECMO. Já os comentários sobre as preocupações com a tolerância da NE foram: "distensão abdominal", "vômito" e "disfunção abdominal"19.

Contudo, outros fatores podem ser apontados no que diz respeito às adequações insuficientes de calorias e proteínas, como a gravidade do quadro clínico na admissão do paciente e uso de sedativos, como propofol. Um estudo observou associação entre inadequação no consumo de proteínas (< $80 \%$ da meta) e maiores valores de escore APACHE II (Acute Physiology and Chronic Health Evaluation II) na admissão. Houve, também, associação entre subnutrição calórica e maiores valores de escore SOFA (Sequential Organ Failure Assessment), no primeiro dia do paciente em ECMO W. Com relação à NE precoce, o tempo para iniciar o suporte nutricional não influenciou no fornecimento adequado de calorias e proteínas neste estudo ${ }^{14}$. Um outro fator pode ser a contribuição calórica de alguns medicamentos que influencia na nutrição. A sedação, por exemplo, é necessária especialmente em pacientes em ECMO W, para otimizar a ventilação e minimizar o consumo de oxigênio. $O$ sedativo comumente utilizado é o propofol, iá mencionado, que contém calorias devido à presença de lipídeos na sua fórmula. Foi relatada uma diferença significativa na média de calorias advindas do propofol em pacientes em ECMO VA $(70 \pm 103 \mathrm{kcal}) \mathrm{e}$ ECMO W (135 $\pm 129 \mathrm{kcal} ; \mathrm{p}<0,001)$, sendo observado o fornecimento de mais de $400 \mathrm{kcal}$ em $24 \mathrm{~h}$ a $37,2 \%$ da amostra total. Desta forma, quando os pacientes toleravam a nutrição na meta e recebiam quantidades significativas de propofol, a fim de evitar o consumo calórico excessivo de calorias era realizada uma redução da nutrição dos pacientes, cujo propofol fornecia $>250 \mathrm{kcal} /$ dia ou $15 \% \mathrm{da}$ necessidade calórica total. A redução da nutrição permite a adequação das calorias, mas, por outro lado, ocasiona o consumo insuficiente de proteínas. Foi observado que a ingestão diária de proteínas nas duas primeiras semanas em pacientes em ECMO WV foi relativamente menor do que a ingestão calórica, sendo associado às características da fórmula nutricional e ao alto volume de propofol que fornece calorias, mas não proteínas. Pacientes com doses $>100 \mathrm{ml} /$ dia de propofol receberam, em média, 65\% da meta proteica, enquanto os demais receberam $76 \%$ da meta $(p=0,03)$. Também foi demonstrado que essa maior necessidade de sedação nos primeiros dias não afetou significativamente a tolerância alimentar, a obtenção da meta ou o tempo até o primeiro movimento intestinal ${ }^{13}$.

A importância da adequação nutricional (>80\%) para os pacientes em ECMO foi demonstrada em estudos que observaram associação com a menor taxa de mortalidade nos dois modos de ECMO $(p=0,01)^{16}$, de $15 \%$ a $39 \%$, enquanto pacientes com inadequação nutricional $(<80 \%)$ apresentaram taxas entre $56 \%$ e $66 \%(p<0,05)^{13,16}$. Por outra ótica, ao avaliar sobreviventes e não sobreviventes, foi observada maior ingestão calórica total entre os sobreviventes $(p=0,037)^{16}$. Contudo, um outro estudo com pacientes em ECMO WV não observou diferença na mortalidade, que foi atribuída a diferenças na gravidade da doença ou a doença subjacente, choque cardiogênico comparado à síndrome do desconforto respiratório agudo, entre os modos de $\mathrm{ECMO}^{14}$. Curiosamente, a permanência no hospital e o tempo em ECMO foram maiores nestes pacientes com nutrição adequada $(>80 \%)^{13,14,16}$. Uma justificativa plausível é que esses pacientes podem tornar-se mais estáveis clinicamente e exigirem menos procedimentos terapêuticos e diagnósticos, permitindo melhor nutrição ${ }^{14}$.

Com relação ao tempo para atingir a meta das necessidades nutricionais, um estudo relatou a obtenção da meta em 2,6 $\pm 1,4$ dias (ECMO W e VA) ${ }^{13}$, período inferior a outro estudo em que os pacientes atingiram a meta calórica após 7,2 $\pm 7,6$ dias $^{16}$. Os demais estudos observaram obtenção entre $50 \%$ e $80 \%$ da meta na primeira semana, incluindo estudo que avaliou pacientes com insuficiência hemodinâmica grave ${ }^{12,15,18}$. Também foi relatado que o tempo para obtenção da meta pode variar entre os modos de ECMO. Conforme observado, o tempo para atingir a meta nutricional foi maior nos pacientes em ECMO VA $(4,0$ $\pm 1,4$ dias) quando comparados aos pacientes em ECMO $\mathrm{W}(2,2 \pm 1,0 \text { dias; } p<0,001)^{13}$. Considerando a recomendação da DGEM ${ }^{11}$, deve-se iniciar a ingestão de calorias com $75 \%$ da meta calórica e aumentar progressivamente de acordo com a tolerância do paciente, atingindo 100\% da meta até o final da fase aguda, que compreende do $4^{\circ}$ ao $7^{\circ}$ dia após início de doença grave. A ESPEN ${ }^{10}$ recomenda que uma dieta hipocalórica pode ser utilizada na fase inicial da doença aguda (não superior a $70 \%$ do gasto energético) e, após $\circ 3^{\circ}$ dia, as calorias devem ser aumentadas para atingir de $80 \%$ a $100 \%$ do gasto energético.

No que se refere aos modos de ECMO, os dados são divergentes, um estudo não observou diferença entre a adequação nutricional dos pacientes em ECMO $\mathrm{W}$ e VA ${ }^{19}$, enquanto outro relatou uma tendência estatística $(p=0,052)$ a diferentes adequações, ambas insuficientes, sendo $67 \%$ nos pacientes em ECMO W e 50\% nos pacientes em ECMO VA. 
O motivo não foi esclarecido, entre as hipóteses estão as diferenças no tipo e quantidade de vasopressores, que não foram registradas, já que não houve diferenças na NP e na administração de drogas procinéticas ${ }^{12}$.

\section{Disfunção Gastrointestinal}

A disfunção gastrointestinal está associada a pacientes em ECMO, conforme mencionado atinge entre $22 \%$ e $22,8 \%$, sendo considerada a segunda causa mais frequente de interrupções da $\mathrm{NE}^{12,14,19}$. A avaliação da disfunção gastrointestinal pode ser feita pela medida do VRG, importante para identificar intolerância à $\mathrm{NE}^{10}$, mas também consideradas a presença de vômito e a distensão abdominal ${ }^{13}$. Altos VRG e a baixa motilidade gástrica são significativos, podendo justificar a melhor adequação nutricional (71\%) após a remoção da ECMO (VA e W) quando comparado ao período em ECMO $(55 \%)^{12}$. Ao analisar, separadamente, outro estudo observou mediana de adequação calórica de $89,8 \%$ em ECMO vs. $93,4 \%$ após a remoção $(p=0,05)$ e adequação proteica de $84,7 \%$ em ECMO vs. $91,2 \%$ pós-ECMO ( $p=0,014)$. Ainda não está claro na literatura se essa dismotilidade gastrointestinal é um resultado da gravidade da doença ou um efeito associado à ECMO. Com a recuperação gradual da doença e a melhora da função gastrointestinal, a adequação nutricional atinge melhores valores ${ }^{14}$.

As diretrizes sugerem que a NE seja adiada quando o VRG for $>500 \mathrm{~mL} / 6 \mathrm{~h}$ sendo, portanto, uma contraindicação à $N E^{10,11}$. Nesta situação, se o exame físico não sugerir uma complicação abdominal aguda, a aplicação de droga procinética deve ser considerada ${ }^{10}$. De fato, conforme já mencionado, o uso das drogas procinéticas foi considerado a primeira alternativa pelos estudos, nos casos de altos VRG dos pacientes em ECMO, na persistência da disfunção gastrointestinal, considerava-se a mudança da posição da sonda para jejunal e, como última alternativa, na ausência de melhora da intolerância, considerava-se o uso de NP12. ${ }^{14}$. Os procinéticos foram utilizados entre $23,3 \%$ a $75 \%$ dos estudos $^{12-15,18,19}$. O uso de sonda na posição pós-pilórica foi proporcionalmente inferior, entre $6 \%$ a $34,5 \%$ dos pacientes $\mathrm{s}^{12,14}$ e realizado em $10 \%$ dos dias nos pacientes em ECMO VA e $\mathrm{V}^{11}$. O uso de NP suplementar foi entre $20,9 \%$ e $30,8 \%$ dos pacientes ${ }^{12,13,15}$, realizado em média $7 \%$ dos dias $^{19}$. Além das medidas mencionadas, uma medida muito simples pode contribuir para VRG menores: a elevação da cabeceira da cama, devido à melhor absorção da $\mathrm{NE}^{12}$. Houve o relato da elevação da cabeceira entre $15^{\circ}$ e $30^{\circ 15,18}$.

Contudo, os estudos consideram valores de VRG diferentes daquele recomendado pelas diretrizes (> $500 \mathrm{~mL} / 6$ h) ${ }^{10,11}$, para identificar disfunção gastrointestinal e considerar o uso de drogas procinéticas. Os protocolos dos estudos consideram verificar o VRG a cada $4 h^{12,14,15}$, havendo o relato de protocolo diferente em um estudo, cujo VRG era verificado a cada $6 \mathrm{~h}$, no $1^{\circ}$ dia, a cada $12 \mathrm{~h}$, no $2^{\circ}$ dia, e diariamente, após $\circ 3^{\circ} \mathrm{dia}^{18}$. Os valores de VRG adotados nos estudos para identificação da intolerância variavam entre $>150$ a $>300 \mathrm{ml}^{11-15,18}$, sendo os valores mais mencionados $>200$ $\mathrm{ml}^{12,13}$. Os estudos também relataram que o VRG elevado deveria estar aumentado em duas ou mais medições ${ }^{13-15} \mathrm{e}$ estar associado à presença de distensão abdominal ${ }^{13}$.

\section{Nutrição e Complicações da ECMO}

\section{Formação de Coágulos}

A formação de coágulos no sistema é considerada a principal complicação da $\mathrm{ECMO}$, sendo um grande desafio estabelecer o equilíbrio entre homeostasia e trombose. Por isso, deve ser realizada diariamente a monitorização clínica e laboratorial dos fatores de coagulação ${ }^{35,36}$. Com relação à nutrição, estudo demonstrou evidências clínicas e in vitro de que o uso combinado de emulsão lipídica intravenosa e ECMO pode estar associado à deposição de gordura nos circuitos ECMO VA e aumento da formação de coágulos sanguíneos ${ }^{37}$. Neste sentido, além da preocupação com a quantidade de lipídeos infundidos diariamente (máximo 1,5 $\mathrm{g} / \mathrm{kg} / \mathrm{dia}$ ), os pacientes em ECMO devem ter uma infusão contínua das emulsões lipídicas por $12 \mathrm{~h}$ e 24 h, sem aplicação em bolus. Além disso, as emulsões lipídicas não devem ser infundidas diretamente no circuito da ECMO e sim por meio de uma linha venosa central remota. A função do oxigenador de membrana deve ser monitorada de perto (oxigenador coagulação) ${ }^{11}$.

\section{Lesão Renal Aguda}

Complicações podem ocorrer durante o manejo do paciente em ECMO, entre elas está a lesão renal aguda (LRA), uma complicação comum decorrente de diversos mecanismos prejudiciais, entre eles a inflamação subjacente, hipoperfusão e exposição a nefrotoxinas. A terapia de substituição renal (TSR) é utilizada em $9 \%$ dos pacientes com insuficiência respiratória e 12\% dos pacientes com insuficiência cardíaca ${ }^{38,39}$. Apesar de ser uma complicação comum, somente um estudo referiu sua experiência com relação à necessidade proteica no uso de terapia de substituição renal contínua (TSRC). Foram utilizados de 1,5 a $2 \mathrm{~g} /$ $\mathrm{kg}$ de proteínas por dia ${ }^{12}$. Houve, também, o relato do início significativamente tardio da nutrição e a presença de mais intolerância nestes pacientes ${ }^{13}$.

\section{CONSIDERAÇÕES FINAIS}

Em resumo, apesar do uso da calorimetria indireta ser viável, não há dados suficientes para a recomendação do uso na prática clínica, devendo considerar a utilização de 
fórmulas baseadas no peso para estimar a necessidade energética destes pacientes. Na determinação das necessidades nutricionais, os estudos utilizaram formas muito distintas para estimar a necessidade calórica, havendo a necessidade de maior padronização, de acordo com as recomendações das diretrizes, pois o gasto energético de pacientes graves em ECMO e sem ECMO não diferiu. Com relação às proteínas, os valores utilizados são semelhantes às recomendações das sociedades de especialidade, porém a escolha da fórmula é determinante na obtenção da adequação nutricional. A NE foi extensamente utilizada nos estudos e demonstrou ser segura e bem tolerada. $O$ início precoce da NE também foi considerado seguro, não sendo atribuído a eventos adversos graves. Quanto aos tipos de fórmula enteral, os estudos divergiram muito, havendo a necessidade de investigações aprofundadas. Uma preocupação específica à ECMO é a necessidade de restrição hídrica, especialmente nos pacientes em ECMO WV que afeta a nutrição, exigindo o uso de fórmulas com baixo volume. Além disso, o uso de medicamentos como o propofol influenciam na escolha da fórmula, por fornecer calorias que devem ser computadas. A via inicial de administração da NE pode ser a gástrica, apesar da ocorrência frequente de disfunção gastrointestinal, avaliada pela presença de altos VRG, sendo considerada a segunda causa mais frequente de interrupções da NE. Os valores de VRG utilizados nos estudos, em geral, são menores do que a recomendação das diretrizes. Nos casos de altos VRG, há a indicação do uso de agentes procinéticos, na persistência da disfunção, considera-se a mudança da posição da sonda para jejunal e, como última alternativa, na ausência de melhora da intolerância, considera-se o uso de NP. O uso da NP suplementar em conjunto com a NE é uma estratégia importante, porém exige um cuidado especial com a administração de glicose e gorduras. Estas estratégias permitem a obtenção da adequação nutricional (>80\%), os estudos demonstram que, apesar da subnutrição ser comum, as metas nutricionais podem ser atingidas. A adequação nutricional é importante a estes pacientes, pela associação com a menor taxa de mortalidade.

Como limitação devemos considerar que, apesar dos dados em concordância, o delineamento da maioria dos estudos é observacional retrospectivo, o que impede uma demonstração de causalidade. Outro fator importante é o fato da maioria dos estudos ter ocorrido em um único centro e a presença de amostras desproporcionais de pacientes nos dois modos de ECMO. Além disso, houve a ausência de distinção nos resultados entre os modos de ECMO (WV e VA) nos estudos incluídos, pois estes compararam o início precoce vs. tardio da NE, sobreviventes vs. não sobreviventes, nutrição adequada vs. nutrição inadequada, mas ainda há muitas dúvidas nos cuidados nutricionais de acordo com o modo de ECMO.
Como perspectivas futuras espera-se a realização de mais estudos prospectivos multicêntricos, que investiguem diferenças entre os cuidados nutricionais em ECMO W e VA, que avaliem as necessidades nutricionais para o estabelecimento de uma recomendação específica a estes pacientes, especialmente calórica e proteica, embora o conhecimento sobre as necessidades de micronutrientes também seja desconhecida. Também serão importantes investigações futuras sobre tipos de dieta que causem menos intolerância e do manejo adequado do VRG para iniciar o uso de drogas procinéticas. Estes detalhes, se avaliados a médio e longo prazo, poderão auxiliar na determinação de recomendações específicas a estes pacientes, visando à sobrevida maior com qualidade de vida.

\section{REFERÊNCIAS}

1. Ribeiro GCA, Succi GM. ECMO: oxigenação por membrana extracorpórea. In: Knobel E, ed. Condutas no paciente grave. $4^{\mathrm{a}}$ ed. São Paulo: Atheneu; 2016. p.531-8.

2. Park M, Azevedo LCP, Mendes PV, Carvalho CRR, Amato MBP, Schettino GPP, et al. First-year experience of a Brazilian tertiary medical center in supporting severely ill patients using extracorporeal membrane oxygenation. Clinics (Sao Paulo). 2012;67(10):1157-63.

3. Chaves RCF, Rabello Filho R, Timenetsky KT, Moreira FT, Vilanova LCS, Bravim BA, et al. Oxigenação por membrana extracorpórea: revisão da literatura. Rev Bras Ter Intensiva. 2019;31(3):410-24.

4. Extracorporeal Life Support Organization(ELSO). International summary. Ann Arbor: Extracorporeal Life Support Organization; 2019. [cited 2020 May 8]. Available from: https://www. elso.org/Registry/Statistics/InternationalSummary.aspx

5. Park M, Costa ELV, Maciel AT, Silva DPE, Friedrich N, Barbosa EVS, et al. Determinants of oxygen and carbon dioxide transfer during extracorporeal membrane oxygenation in an experimental model of multiple organ dysfunction syndrome. PLoS One. 2013;8(1):e54954.

6. Alberda C, Gramlich L, Jones N, Jeejeebhoy K, Day AG, Dhaliwal $\mathrm{R}$, et al. The relationship between nutritional intake and clinical outcomes in critically ill patients: results of an international multicenter observational study. Intensive Care Med. 2009;35(10):1728-37.

7. Farías MM, Olivos C, Díaz R. Nutritional implications for the patient undergoing extracorporeal membrane oxygenation. Nutr Hosp. 2015;31(6):2346-51.

8. Bear DE, Smith E, Barrett NA. Nutrition support in adult patients receiving extracorporeal membrane oxygenation. Nutr Clin Pract. 2018;33(6):738-46.

9. Zonies D, Codner P, Park P, Martin ND, Lissauer M, Evans S, et al. AAST Critical Care Committee clinical consensus: ECMO, nutrition. Trauma Surg Acute Care Open. 2019;4(1):e000304.

10. Singer P, BlaserAR, Berger MM, Alhazzani W, Calder PC, Casaer $\mathrm{MP}$, et al. ESPEN guideline on clinical nutrition in the intensive care unit. Clin Nutr. 2019;38(1):48-79.

11. Elke G, Hartl WH, Kreymann KG, Adolph M, Felbinger TW, Graf $\mathrm{T}$, et al. Clinical nutrition in critical care medicine - guideline of the German Society for Nutritional Medicine (DGEM). Clin Nutr ESPEN. 2019;33:220-75.

12. Lukas G, Davies AR, Hilton AK, Pellegrino VA, Scheinkestel CD, Ridley E. Nutritional support in adult patients receiving extracorporeal membrane oxygenation. Crit Care Resusc. 2010;12(4):230-4. 
13. Ferrie S, Herkes R, Forrest P. Nutrition support during extracorporeal membrane oxygenation (ECMO) in adults: a retrospective audit of 86 patients. Intensive Care Med. 2013;39(11):1989-94.

14. MacGowan L, Smith E, Elliott-Hammond C, Sanderson B, Ong $\mathrm{D}$, Daly K, et al. Adequacy of nutrition support during extracorporeal membrane oxygenation. Clin Nutr. 2019;38(1):324-31.

15. Scott LK, Boudreaux K, Thaljeh F, Grier LR, Conrad SA. Early enteral feedings in adults receiving venovenous extracorporeal membrane oxygenation. JPEN J Parenter Enteral Nutr. 2004;28(5):295-300.

16. Lu MC, Yang MD, Li PC, Fang HY, Huang HY, Chan YC, et al. Effects of nutritional intervention on the survival of patients with cardiopulmonary failure undergoing extracorporeal membrane oxygenation therapy. In Vivo. 2018;32(4):829-34.

17. Ohbe H, Jo T, Yamana H, Matsui H, Fushimi K, Yasunaga H. Early enteral nutrition for cardiogenic or obstructive shock requiring venoarterial extracorporeal membrane oxygenation: a nationwide inpatient database study. Intensive Care Med. 2018;44(8):1258-65.

18. Umezawa Makikado LD, Flordelís Lasierra JL, Pérez-Vela JL, Colino Gómez L, Torres Sánchez E, Maroto Rodríguez B, et al. Early enteral nutrition in adults receiving venoarterial extracorporeal membrane oxygenation: an observational case series. JPEN J Parenter Enteral Nutr. 2013;37(2):281-4.

19. Ridley EJ, Davies AR, Robins EJ, Lukas G, Bailey MJ, Fraser JF; Australian and New Zealand Extracorporeal Membrane Oxygenation Nutrition Therapy. Nutrition therapy in adult patients receiving extracorporeal membrane oxygenation: a prospective, multicentre, observational study. Crit Care Resusc. 2015;17(3):183-9.

20. Weir JB. New methods for calculating metabolic rate with special reference to protein metabolism. J Physiol. 1949;109(1-2):1-9.

21. Avesani CM, Santos NSJS, Cuppari L. Necessidades e recomendações de energia. In: Cuppari L, ed. Nutrição clínica no adulto. $3^{\mathrm{a}}$ ed. Barueri: Manole; 2014. p.41-57.

22. De Waele E, van Zwam K, Mattens S, Staessens K, Diltoer M, Honore PM, et al. Measuring resting energy expenditure during extracorporeal membrane oxygenation: preliminary clinical experience with a proposed theoretical model. Acta Anaesthesiol Scand. 2015;59(10):1296-302.

23. Wollersheim T, Frank S, Müller MC, Skrypnikov V, Carbon NM, Pickerodt PA, et al. Measuring Energy Expenditure in Extracorporeal Lung Support Patients (MEEP): protocol, feasibility and pilot trial. Clin Nutr. 2018;37(1):301-7.

24. De Waele E, Jonckheer J, Pen JJ, Demol J, Staessens K, Puis L, et al. Energy expenditure of patients on ECMO: a prospective pilot study. Acta Anaesthesiol Scand. 2019;63(3):360-4.

25. Schofield WN. Predicting basal metabolic rate, new standards and review of previous work. Hum Nutr Clin Nutr. 1985;39(Suppl 1): $5-41$.

26. Cerra FB, Benitez MR, Blackburn GL, Irwin RS, Jeejeebhoy K, Katz DP, et al. Applied nutrition in ICU patients. A consensus statement of the American College of Chest Physicians. Chest. 1997;111(3):769-78.
27. Harris JA, Benedict FG. A biometric study of human basal metabolism. Proc Natl Acad Sci USA. 1918;4(12):370-3.

28. McClave SA, Taylor BE, Martindale RG, Warren MM, Johnson $\mathrm{DR}$, Braunschweig $\mathrm{C}$, et al. Guidelines for the provision and assessment of nutrition support therapy in the adult critically ill patient: Society of Critical Care Medicine (SCCM) and American Society for Parenteral and Enteral Nutrition (A.S.P.E.N.). JPEN J Parenter Enteral Nutr. 2016;40(2):159-211.

29. Pelekhaty S, Galvagno SM Jr, Hochberg E, Herr DL, Lantry JH, Kon $\mathrm{ZN}$, et al. Nitrogen balance during venovenous extracorporeal membrane oxygenation support: preliminary results of a prospective, observational study. JPEN J Parenter Enteral Nutr. 2020;44(3):548-53.

30. Koning NJ, Vonk ABA, van Barneveld LJ, Beishuizen A, Atasever B, van den Brom CE, et al. Pulsatile flow during cardiopulmonary bypass preserves postoperative microcirculatory perfusion irrespective of systemic hemodynamics. J Appl Physiol. 2012;112(10):1727-34.

31. Kurundkar AR, Killingsworth CR, Mcllwain RB, Timpa JG, Hartman YE, He D, et al. Extracorporeal membrane oxygenation causes loss of intestinal epithelial barrier in the newborn piglet. Ped Res. 2010;68(2):128-33.

32. Kagan I, Singer P. Nutritional imbalances during extracorporeal life support. World Rev Nutr Diet. 2013;105:154-9.

33. Wells DL. Provision of enteral nutrition during vasopressor therapy for hemodynamic instability: an evidence-based review. Nutr Clin Pract. 2012;27(4):521-6.

34. Marvin RG, McKinley BA, McQuiggan M, Cocanour CS, Moore FA. Nonocclusive bowel necrosis occurring in critically ill trauma patients receiving enteral nutrition manifests no reliable clinical signs for early detection. Am J Surg. 2000;179(1):7-12.

35. Lubnow M, Philipp A, Foltan M, Bull Enger T, Lunz D, Bein $\mathrm{T}$, et al. Technical complications during veno-venous extracorporeal membrane oxygenation and their relevance predicting a system-exchange: retrospective analysis of 265 cases. PloS One. 2014;9(12):e112316.

36. Shekar K, Roberts JA, Barnett AG, Diab S, Wallis SC, Fung YL, et al. Can physicochemical properties of antimicrobials be used to predict their pharmacokinetics during extracorporeal membrane oxygenation? Illustrative data from ovine models. Crit Care. 2015;19:437.

37. Lee HM, Archer JR, Dargan PI, Wood DM. What are the adverse effects associated with the combined use of intravenous lipid emulsion and extracorporeal membrane oxygenation in the poisoned patient? Clin Toxicol. 2015;53(3):145-50.

38. Thiagarajan RR, Barbaro RP, Rycus PT, McMullan DM, Conrad SA, Fortenberry JD, et al. Extracorporeal Life Support Organization registry international report 2016. ASAIO J. 2017;63(1):60-7.

39. Ostermann M, Connor M Jr, Kashani K. Continuous renal replacement therapy during extracorporeal membrane oxygenation: why, when and how? Curr Opin Crit Care. 2018;24(6):493-503.

Local de realização do estudo: Hospital Israelita Albert Einstein, São Paulo, SP, Brasil.

Conflito de interesse: Os autores declaram não haver. 